\title{
Drumming with a Humanoid Robot: Results from Human-Robot Interaction Studies
}

\author{
Extended abstract
}

\author{
Hatice Kose-Bagci, Kerstin Dautenhahn, and Chrystopher L. Nehaniv \\ Adaptive Systems Research Group, University of Hertfordshire, School of Computer Science, \\ Hatfield, Herts, AL 10 9AB, U.K. \\ \{h.kose-bagci,k.dautenhahn,c.1.nehaniv\}@herts.ac.uk
}

\begin{abstract}
We summarise two human-robot interaction studies investigating a drumming experience with Kaspar, a humanoid child-sized robot, and human participants. Our aim $^{1}$ is not to have Kaspar just replicate the human's drumming, but to engage in a 'social manner', i.e. in a call and response turn-taking interaction. The interactions are discussed in terms of imitation, turntaking, and the effect of gender differences. This research is part of a project into developmental robotics with a particular emphasis of our work on gesture communication.
\end{abstract}

\section{Introduction}

We present two exploratory studies investigating a drumming experience with Kaspar [1] and human participants. The primary goal of this work is to achieve (non-verbal) gesture communication between child-like humanoid robots and human beings, whereby drumming served as a test bed to study key aspects such as turntaking and non-verbal gestures.

In the first presented study, turn-taking is deterministic and head gestures of the robot accompany its drumming to assess the impact of non-verbal gestures on the interaction [2]. The second study focuses on emergent turn-taking dynamics; here our aim is to have turn-taking and role switching which is not deterministic but emerging from the social interaction between the human and the humanoid [3]. Therefore the robot is not just 'following' and imitating the human, but can be the leader in the game and being imitated by the human. Details of the two studies summarized in this paper as well as related work can be found in $[2,3]$.

\footnotetext{
${ }^{1}$ Acknowledgements: This work was conducted within the EU Integrated Project RobotCub ("Robotic Open-architecture Technology for Cognition, Understanding, and Behaviours"), funded by the EC through the E5 Unit (Cognition) of FP6-IST under Contract FP6-004370.
}

\section{Using gestures as social cues}

In this first study human participants played a rhythm which Kaspar tried to replicate, in a simple form of imitation (mirroring), by hitting the drum positioned in its lap. Then the human partner played again. This (deterministic) turn-taking continued for the fixed duration of the game. Kaspar did not imitate the strength of the beats but only the number of beats and duration between beats, due to its limited motor skills. Our primary focus was to study the possible impact that utilizing social gestures would have, not only on the game itself (in terms of performance), but also on the participant's subsequent subjective evaluation of the game.

We studied three conditions with increasing amounts of gesturing. In the first condition Kaspar did not use any gestures. Kaspar only imitated the drumming. This condition was called no-gesture. In the gesture game, simple head gestures and eye blinking were included in Kaspar's movements. Kaspar started drumming with one of the fixed gestures. If the human partners did not play their turn, then Kaspar as well did not do anything and the turn passed to the partner. In the gesture+ condition, Kaspar simply repeated the sequence of gestures without playing even if the partners did not play their turn.

Tests and results: The 3 conditions with all possible orders were tested with 12 adult participants ( 6 male and 6 female) who worked in computer science or similar disciplines at the University, and were overall not familiar with robots. .

According to the questionnaires, for the least preferred game type, there were significant differences due to gender $\left(\chi^{2}(1,11)=4.75, p=.03\right)$. This difference manifests as males predominantly choosing the gesture + game type as their least preferred game type, while females predominantly chose the no-gesture game type as their least preferred game (Fig. 1).

According to the observed behaviours, although the error rate (describing how well Kaspar can replicate a human's drumming) in gesture+ was less than in the gesture condition, male participants liked it the least overall. In contrast, although the error rate in gesture+ was the highest, female participants liked it more than the 
no-gesture game which had the lowest error rate (Table $1)$.
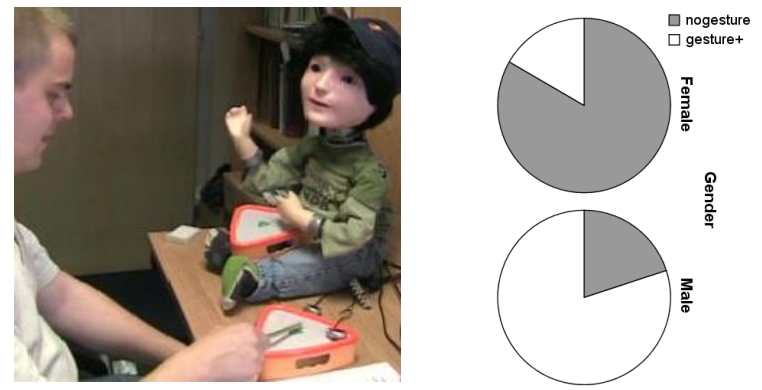

Fig. 1 A screen shot from the experiments (left) . Least preferred game type according to gender (right)

\begin{tabular}{ccc}
\multicolumn{2}{c}{ Table 1 Observed behaviour according to game type } \\
\cline { 2 - 3 } Game type & \multicolumn{2}{c}{ Avg. error } \\
\cline { 2 - 3 } & males & females \\
\hline no-gesture & $2.5 \pm 2.7$ & $2.7 \pm 2.5$ \\
gesture & $3.9 \pm 3.6$ & $3.1 \pm 3.8$ \\
gesture + & $3.0 \pm 2.9$ & $3.5 \pm 3$
\end{tabular}

While an exhaustive description of the qualitative analysis of the participants' responses is beyond the scope of this abstract, the above results are due to differences in task vs. interaction orientation between participants. Male participants tended to be more task oriented, while female participants tended to evaluate the interaction as a whole.

\section{Emergent turn-taking dynamics}

Timing plays a fundamental role in the regulation of human-human interaction. In the first study described above, some predefined fixed time duration heuristics were used for synchronization. Kaspar started playing if the human partner was silent for a few seconds. However, it was not always clear when the robot or human partner should initiate interaction in taking a turn. In this second study, we instead used a probability-based algorithm to control timing and turn-taking. The temporal dynamics of turn-taking thus emerged from the interaction between the human and the humanoid. Three simple models (threshold, linear, hyperbolic) were used to control the starting and stopping of the robot's drumming beats. This response was based on the duration of the previous turn and on the number of beats played in the previous turn by the interaction partners.

Our primary research interests were to study how different robot turn-taking strategies based on computational, probabilistic models impact the drumming performance of the human-robot pair, and the participants' subjective evaluation of the drumming experience. From the results of 12 participants, we observed in terms of subjective evaluation a significant difference between the first and third games in terms of order. Human participants got used to the game as they played more. In terms of observed behaviour, different models gave different amounts of playing time to Kaspar and the human participants, which affected participants' preferences. Most of the participants did not prefer a linear model because it gave them the least playing time, whereby Kaspar ignored them and played on its own which influences social interaction negatively. Some of the participants preferred to replicate Kaspar's drumming, and the "follower" and "leader" roles switched (unlike the first work (section 2), where Kaspar was always a "follower", and humans were always "leaders").

\section{Conclusion}

We presented the result of interaction games with 24 participants. Due to the small participant sample size the analysis is only descriptive.

In the first study Kaspar just repeated the beats produced by the human partner, and made simple fixed head gestures accompanying its drumming. The human partners' in return, perceived these simple behaviours as more complex and meaningful. Note, while Kaspar's drum playing did not change over time, and stayed the same in different games, the participants learned the limits of Kaspar and the rules of the game, and adapted themselves to the game better (future work will investigate this adaptation in more detail), so the success rate improved over time. It seems that participants were not passive subjects in this game, but unconsciously adapted their own behavior to the capabilities of the robot. In the second study involving emergent turn-taking dynamics, although we used very simple models, we were able to observe some very successful games in terms of coordinated turn-taking, and role switching emerging from social interaction between the human and the humanoid. These gesture communication studies can possibly be extended for use in the other robotic fields, e.g. entertainment, service robots, and educational/therapy robots.

\section{Selected References}

[1] M.P. Blow, K. Dautenhahn, A. Appleby, C. Nehaniv, D. Lee, "Perception of robot smiles and dimensions for humanrobot interaction design", In Proc. IEEE RO-MAN06, pp.469474, 2006.

[2] H. Kose-Bagci, K. Dautenhahn, D. S. Syrdal, and C. L. Nehaniv, "Drum-mate: A Human-Humanoid Drumming Experience", IEEE-RAS Humanoids2007, 2007.

[3] H. Kose-Bagci, K. Dautenhahn, and C. L. Nehaniv, "Emergent Turn-Taking Dynamics in Drumming Games with a Humanoid Robot”, IEEE RO-MAN 2008, 2008 - accepted 